\title{
Study on EEG Feature Extraction under LED Color Exposure to Enhance the Concentration
}

\author{
Jung-Eun Lim ${ }^{1,}$ a , Bo-Hyeok Seo ${ }^{2, b}$, Sun Hyun Kim, c, Soon-Yong Chun ${ }^{4, ~ d ~}$ \\ ${ }^{1}$ School of Electrical Engineering and computer Science, The Graduate School, \\ Kyungpook National University, Korea \\ ${ }^{2}$ School of Electrical Engineering and computer Science, Kyungpook National University, Korea \\ ${ }^{3} \mathrm{CHA}$ Medical University, Korea \\ ${ }^{4}$ Dong Yang University, Korea \\ aeuny1122@knu.ac.kr, bbhsuh@knu.ac.kr, 'ckshcat1210@hanmail.net,, ${ }^{d}$ control@dyu.ac.kr
}

\begin{abstract}
Keywords: LED, Color Brain Wave (CBW), Concentration, EEG, Intensive EEG Index
\end{abstract}
\begin{abstract}
Since brain waves are expressed in a variety of frequency domains, they were used to analyze a correlation between colors and concentration. In this study, the brain wave reacting when exposed to colors was defined as a color brain wave (CBW). Also the colors on the table were changed during task performance to see colors' influence on improving concentration and then the brave waves were measured for analysis on and comparison with the findings from the task performance. Based on the biometric data experiment conducted, it was confirmed that the findings during the task performance and those from EEG signals have a correlation and that human's concentration is thus affected by changes of colors.
\end{abstract}

\section{Introduction}

As it becomes known that the bodily organ involved in human's thought, including object cognition and thinking, is the brain and that this brain constitutes a nerve system entangled in a complex form [1], a number of brain science researches on human's cognition and thought are actively proceeding. To raise human's capability to process cognition, various researches related to memory, attention, emotion, and others have been attempted $[2,3,4]$. For example, tennis players were selected to allow them to participate in the training of adjusting their brain waves. As a result of this training, their concentration was enhanced [5]. And it was reported that the meditation using classical music also improved their inner awakening level, raising learners' attention on task performance and their psychological stability [6]. The ultimate purpose of this study is to generate positive brain waves and thus to improve a cognitive capability. Since brain waves are expressed in various frequencies, it is possible to analyze the status of the brain on human's behaviours [7]. Even though a number of preliminary studies are proceeding to figure out a correlation between concentration and brain waves, the studies on neuro - feedback to improve concentration using colors remain insufficient. Thus, this study intended to figure out feature extraction of colors' influence on concentration and their correlation focusing on the LED three primary colors. For the feature extraction on CBW, intensive EEG index was used and an experiment to figure out a correlation between colors and concentration was conducted. Then, percentage correct and performance time were used to compare and analyze measured brain waves.

\section{Color Brain Wave}

Brain waves differ among individuals and the wave forms are also different depending on the subjects' ages, even if these brain waves are normal EEG. Of the brain waves making people keep stable with their eyes closed, not in soul-sleep, the most dominant rhythm is called "basic rhythm." The brain wave's amplitude while eyes are being closed reaches $30 \sim 50 \mu \mathrm{V}$. And in the test's brain wave while he or she is in a stable, awakened, and eye-closed state, alpha rhythm becomes dominant 
in the occipital region. If beta rhythm is mixed in here, it is the fundamental aspect, bilateral synchro (nous pattern), and symmetry [8]. The measured brain wave's amplitude during eye-closed reaches $30 \sim 50 \mu \mathrm{V}$, in which case alpha wave'rhythm showed the fundamental aspect and bilateral synchro (nous pattern) in a symmetry state. The brain's amplitudes when exposed to colors reached approximately $10 \sim 30 \mu \mathrm{V}$, in which case the amplitude's maximum value did not exceed its minimum value in a normal state. In addition, the vitalization level by segment when the brain wave was exposed to colors was different between preferential colors and non-preferential ones, and the symmetry was not maintained depending on vitalized segments. Fig. 1 shows the waveforms measured during eye-closed and in a stable state and those when the brain wave were exposed to colors. The brain wave measured in a state of exposed to colors, compared with normal EEG, was defined as a CBW.

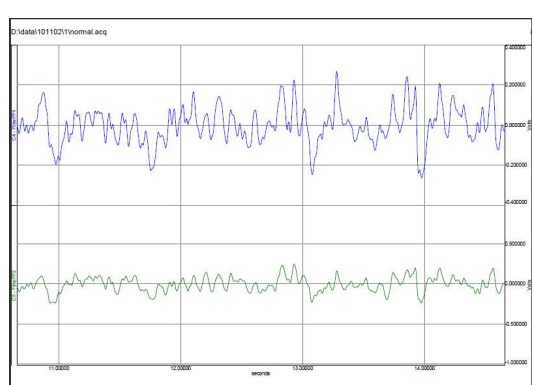

(a)eye-closed state

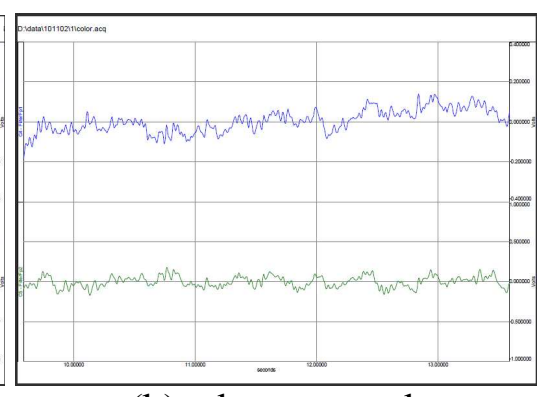

(b)color-exposed state

Fig. 1. Measured brain wave

\section{Concentration}

"Concentration" is defined as an attentive review on a certain stimulus or a state of maintaining attention. "Attention" is defined as the concentration or focus on the consciousness of clearly and vividly keeping in mind by selecting one between the object and the thought [9]. In other words, attentive concentration is a capability to maintain attention to a stimulus. It is known that the EEG areas having a high correlation with concentration are Beta waves, SMR waves, and high Beta waves [10]. In this study, for the feature extraction on CBW, measured waves were classified into 3 areas and an Intensive EEG Index was defined and used. To figure out colors' influence on concentration and their correlation with brain waves, the LED three primary colors were used to measure and analyze the brain wave during task performance on the color table that may change. The color table used in the experiment was $90 \mathrm{~cm}$ in width, $180 \mathrm{~cm}$ in length, and $75 \mathrm{~cm}$ in height, and its top plate was made of the translucent acrylic materials designed to spread the light. In the lower of its top plate, LED was installed to change colors using a controller. Between individual colors, an intermediary was set up. After the test solves problems, the percentage correct, performance time, and data on preferential colors through a survey were used for comparison with and analysis on the values processed using the Intensive EEG Index. As a numerical formula, Eq. 1 was used.

$$
\text { Intensive EEG Index }=(\text { SMR }+ \text { Mid-Beta }) / \text { Theta }
$$

\section{Brain Wave Measurement and Analysis}

The experiment proceeded in a room designed as a structure dark room to block external noises and the light, where temperature and humidity are steadily maintained. There is no electronic device other than the LED (40W) daylight installed on the inner ceiling. The test comes to attend the experiment in state of sitting on a chair. As subjects, 11 men and women over 18 years were selected, who were not color blind with any signs of fatigue and whose bodily functions were normal. Prefrontal lobe segments Fp1, Fp2 are in charge of functions such as connition \& thought, and creativity, playing a central role in terms of learning behaviors [11]. Accordingly, the segments Fp1, Fp2 were applied as a measuring point, and the performance tasks were given the questionnaires that any Korean can 
solve if he or she has finished elementary education courses. They were required to select preferred questionnaires and to find out typing errors after reading them early. Using Biopac MP150 and EEG $100 \mathrm{C}$, brain waves were measured, and using a notch filter, external power noises were removed. For measured signals, MATLAB was used to design a filter that may largely classify theses signals into SMR waves, Mid-Beta waves, and Theta waves. Then, the band to band values were acquired. The signals classified in this way were applied to a concentricity extraction algorithm to extract the Intensive EEG Index. Then, this Intensive EEG Index was used to compare and analyze extracted values and the findings from performing tasks. The color table and the picture while the brave waves were being measured were shown in Fig. 2.

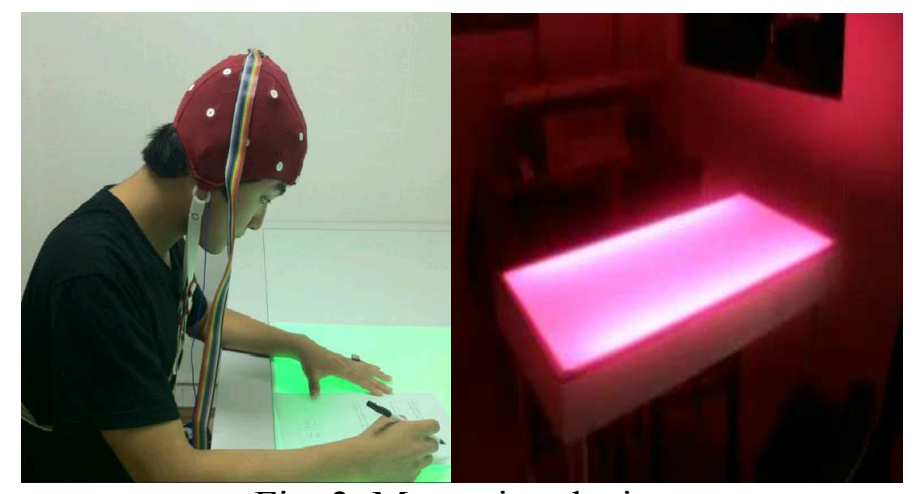

Fig. 2. Measuring device

\section{Result}

Fig. 3 shows the Intensive EEG Indexes by each case in the primary colors: red, green, and blue. Table 1 indicates the percentage correct in each case and the performance time, as well as preferred colors and non-preferred colors.

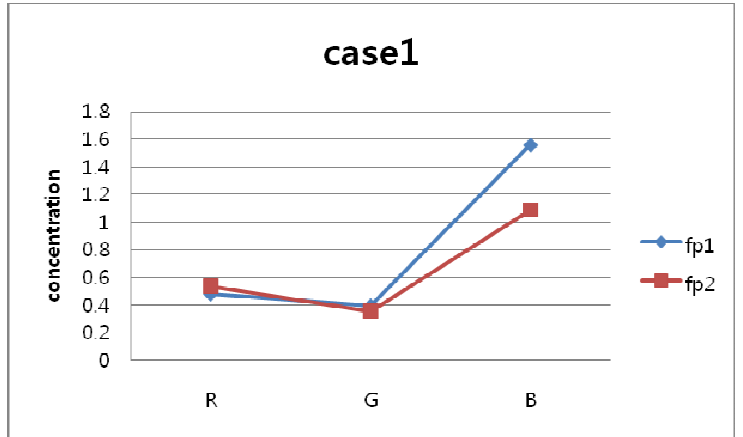

(a) Case 1

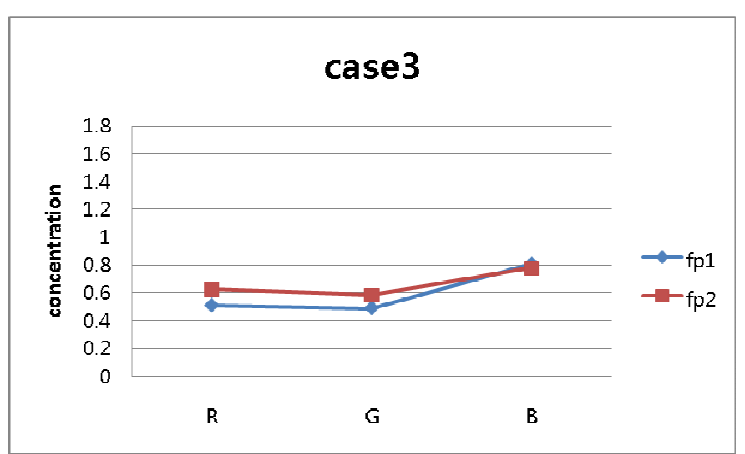

(c) Case 3

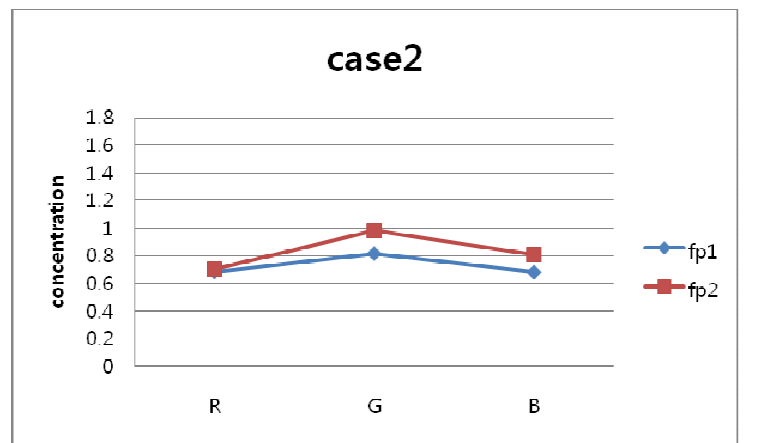

(b) Case 2

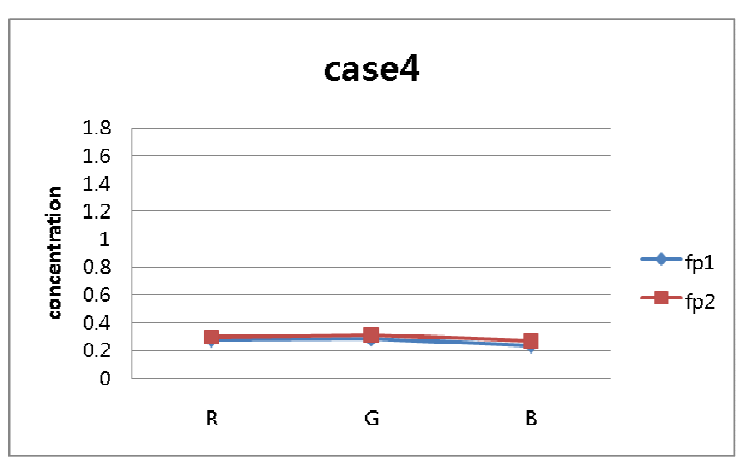

(d) Case 4 


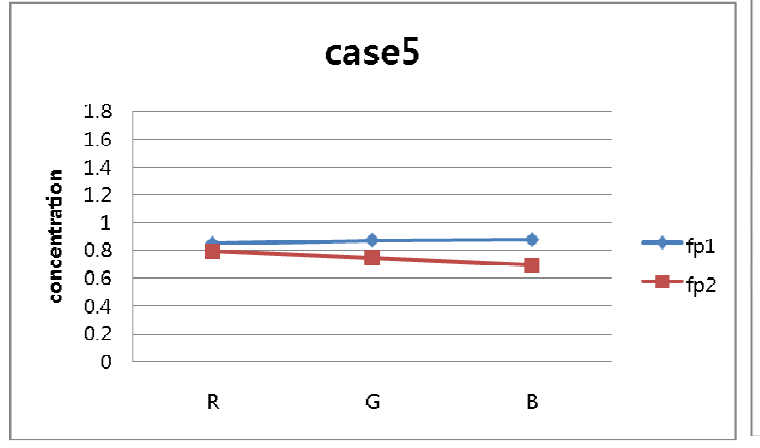

(e) Case 5

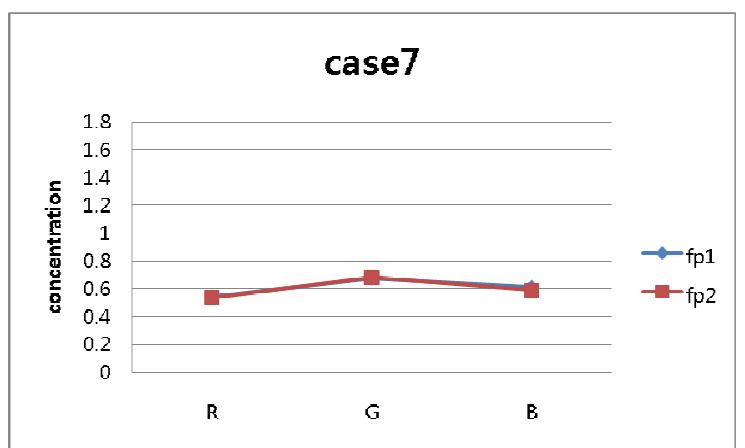

(g) Case 7

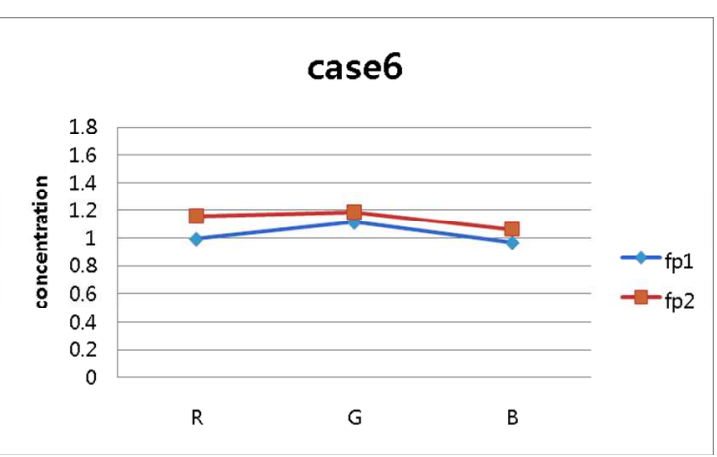

(f) Case 6

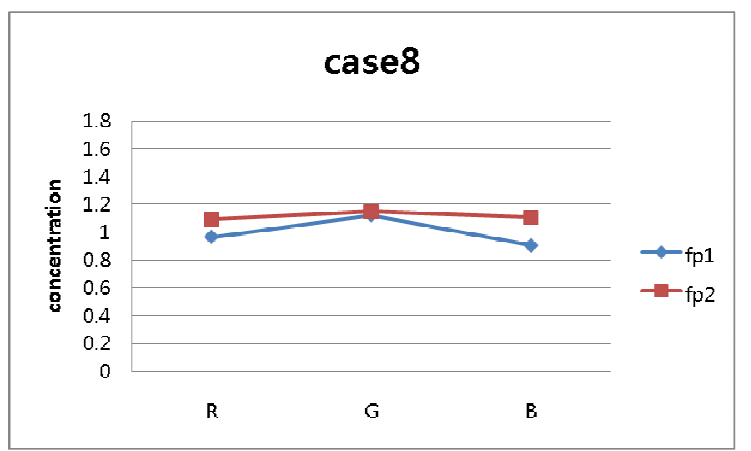

(h) Case 8

Table 1

\begin{tabular}{|c|c|c|c|c|c|c|c|c|}
\hline & \multirow{2}{*}{$\begin{array}{c}\text { Preferred } \\
\text { color }\end{array}$} & \multirow{2}{*}{$\begin{array}{c}\text { Non- } \\
\text { preferred } \\
\text { color }\end{array}$} & \multicolumn{2}{|c|}{ Percentage of correct (\%) } & \multicolumn{3}{|c|}{ Performance time(s) } \\
\cline { 5 - 10 } & & Red & Green & Blue & Red & Green & Blue \\
\hline Case1 & Blue & Green & 85 & 95 & 95 & 41 & 52 & 47 \\
\hline Case2 & Green & Red & 90 & 90 & 90 & 47 & 44 & 48 \\
\hline Case3 & Red & Green & 65 & 50 & 60 & 31 & 33 & 28 \\
\hline Case4 & Green & Blue & 65 & 85 & 65 & 38 & 40 & 41 \\
\hline Case5 & Green & Red & 65 & 70 & 75 & 41 & 40 & 37 \\
\hline Case6 & Green & Red & 75 & 65 & 75 & 33 & 36 & 35 \\
\hline Case7 & Green & Blue & 70 & 75 & 85 & 33 & 29 & 35 \\
\hline Case8 & Green & Red & 70 & 80 & 75 & 37 & 39 & 45 \\
\hline
\end{tabular}

In Case 1, the Intensive EEG Index was higher in green and blue than red, and the percentage correct was also higher. They preferred blue to green. The percentage correct in the three colors was same, but the task performance took longer in green than blue. In Case 2, the Intensive EEG Index was the highest in their preferred color, green. The percentage correct in the three colors was all same, but the task performance took shortest in green. In Case 3, they preferred red, but blue showed the highest in the Intensive EEG Index and its percentage correct also was much higher than that in the green color that they did not prefer. Case 4 showed the highest percentage correct in green that they preferredm but lower perecentage correct and the longest performance time in their non-preferred color, blue. In Case 5, their preferred color, green was higher in the Intensive EEG Index but the longest performance time and lower percentage correct. Case 6 also showed the highest Intensive EEG Index in green that they preferred, as well as higher percentage correct and shorter performance time. In Case 6 also, the Intensive EEG Index was high in green, and the percentage correct was high along with short performance time. In Case 7 also, green that they preferred the highest Intensive EEG 
Index and the shortest performance time. Case 8 as well showed the highest Intensive EEG Index in green and the highest percentage correct. It was observed that their preferred color showed the highest percentage correct and their non-preferred color indicated the lower value. Percentage correct and performance time also seem to have been affected by their preferred or non-preferred color. In addition, bluish colors showed higher value in the percentage correct than reddish colors.

\section{Conclusion}

This study was conducted to figure out colors' influence on concentration. For this, the color on the table was changed during task performance and then the brain waves were measured. Then the measured brain waves were used to make an comparision with the findings from performing tasks, and these data were analized. The brain wave that responded when colors were exposed was defined as a CBW. It was confirmed through the conducted biometrics test that general brain waves and CBW are diffrent. The tasks considered to allow the subjects to concentrate were performed and their precentage correct, performance time, and EEG signals were collected. The collected EEG signals were transformed into frequency domains and then the EEG areas related to concentration were classfied. And the Intensive EEG Indexes were analized using a concentration extraction formula. As a result of that, it was obeserved that the case when the sujects solved problems using the colors on the top plate of the color table, showing the higher Intensive EEG Index, indicated higher percentage correct and shorter performance time than the rest cases. Individually, preferred colors showed much higher values in the Intensive EEG Index than non-preferred colors. In addition, it was found out that they prefer blue colors on the whole and that bluish colors show higher values in the Intensive EEG Index than reddish colors. In conclusion, human's coconcentration is affected by colors. Thus, if the findings from this study are used to improve concentration, it would be possible to lay a foundation to get better results in solving problems, especially dealing with computational problems.

\section{References}

[1] F. Crick, The Astonishing Hypothesis: The scientific Search for the Soul, Contemporary psychology, Vol. 41 No. 5 (1996) 427-428

[2] Robbins, J., Wired for miracles (neurofeedback therapy). Psychology Today, May 1th

[3] Anna, W., High performance mind, New York, Tarcher Putnam.1995

[4] Nak, C. L., Correlates of eeg hemispheric integration. Ph. D. Indiana University.1992

[5] Jang, Effects of concentration training with brainwave biofeedback on tennis performance, Seoul University, 2001

[6] Reitman, A., A pilot stydt: The effect of music-assisted coping systematic desensitization on music performance anxiety: A three-system model approach, Temple University, 1997

[7] Kim, Sung woon, The effect of eeg bio-feedback training on attention and brain activity in moderate mental retardation, journal of intellectual disabilities, 2009, Vol. 11, No. 4, pp. $117-136$

[8] Kim, Electroencephalogram, Seoul: pp.51-67, 2001

[9] Sterman, M. B., Sensorimotor EEG operant conditioning and experimental and clinical effects. Pavlovian J. Biological Science, 12(2), pp.65-92, 1977

[10] Jame, W., The principle of psychology, Vol. 1. Holt: NY, 1890

[11] A. E. Lawson., Therole of the prefrontal lobes in scientific reasoning, The Korea Association for Science Education, 17(4), 525-540, 1997 